\title{
On the thermal and thermomechanical assessment of the "Optimized Conservative” Helium-Cooled Lithium Lead Breeding Blanket concept for DEMO
}

\author{
G. Bongiovì , G. Aiello ${ }^{\mathrm{b}}$, J. Aubert ${ }^{\mathrm{b}}$, P. A. Di Maio ${ }^{\mathrm{a}}$ \\ ${ }^{a}$ Dipartimento di Energia, Ingegneria dell'Informazione e Modelli Matematici, \\ Università di Palermo, Viale delle Scienze, I-90128 Palermo, ITALY \\ ${ }^{b}$ CEA Saclay, DEN, DM2S, 91191, Gif-Sur-Yvette, FRANCE
}

\begin{abstract}
Within the framework of EUROfusion R\&D activities a research campaign has been performed at CEA-Saclay, in close collaboration with the University of Palermo, in order to investigate thermal and thermomechanical performances of the "Optimized Conservative" concept of DEMO Helium-Cooled Lithium Lead breeding blanket (HCLL). Attention has been paid to the HCLL outboard equatorial module (OEM) when subjected to the steady state nominal loading scenario. To this purpose three simplified 3D models, characterized by an increasing level of detail, have been set-up taking into account, firstly, a single radial-toroidal slice, then a basic module geometric unity composed by two adjacent slices and adding, lastly, the peripheral poloidal region. This latter 3D model has allowed the assessment of the Caps potential influence on the module thermal and thermomechanical behaviour. For each investigated 3D model, thermal and thermomechanical analyses have been performed and a stress linearization procedure has been carried out in order to verify the fulfilment of the criteria prescribed by the RCCMRx 2015 code. The study has been performed adopting a numerical approach, based on the Finite Element Method (FEM), and adopting the Siemens NX v. 10.0 software in order to discretize the geometric domain, whereas thermal and thermomechanical calculations have been carried out using the Cast3M 2015 FEM code.

The obtained results, herewith reported and critically discussed, allow predicting a good thermal and mechanical behaviour of the "Optimized Conservative" concept of DEMO HCLL OEM, even if some small modifications to the module cooling scheme should be performed in order to avoid the insurgence of hotspots where temperature is slightly above the Eurofer limit temperature $\left(550^{\circ} \mathrm{C}\right)$. This will entail, from the mechanical point of view, a reduction of the secondary stress amount which is the main responsible of the failure in RCC-MRx criteria verification within First Wall-Side Wall bend region.
\end{abstract}

Keywords: DEMO, HCLL, breeding blanket, thermomechanics, FEM analysis

\section{Introduction}

Within the framework of EUROfusion activities, CEA Saclay is in charge of developing the design of the Helium-Cooled Lithium Lead breeding blanket (HCLL) of DEMO nuclear fusion reactor.

In this context a fruitful collaboration has taken place with University of Palermo, as this latter has been involved, long time now, in the studies regarding the ITER Test Blanket Module (TBM) based on HCCL concept [1].

The work described in this paper, developed within the above said collaboration, has been aimed at the investigation of the thermal and thermomechanical performances of the HCLL Outboard Equatorial Module (OEM) under the envisaged nominal steady state loading conditions, paying also attention to the automation of the analysis procedure. In particular, the study has been aimed at the verification of the fulfilling of the set of thermomechanical requirements, in accordance with RCC-MRx code [2], prescribed for HCLL design.

A theoretical-numerical approach, based on the Finite Element Method (FEM), has been followed and the qualified Cast3M 2015 and Siemens NX v. 10.0 FEM codes $[3,4]$ have been adopted in the study.

\section{HCLL “Optimized Conservative” concept}

Within the framework of HCLL design activities [5], 3 different concepts (Optimized Conservative, Advanced [6,7], Advanced-Plus [8]) have been assessed. All the concepts use Eurofer steel as structural material, Helium at the pressure of $8 \mathrm{MPa}$ as coolant and the eutectic alloy $\mathrm{Pb}-15.7 \mathrm{Li}$ enriched at $90 \%$ in $6 \mathrm{Li}$ as breeder, neutron multiplier and tritium carrier.

In this paper, attention has been focused on the "Optimized Conservative” concept, which is consistent with the TBM approach [9] and is considered as backup solution for DEMO HCLL [5].

In this concept (Fig. 1), the First Wall (FW) is the component directly exposed to plasma radiation. Its thickness is $25 \mathrm{~mm}$ plus $2 \mathrm{~mm}$ of tungsten coating, whereas cooling is ensured by means of internal channels. The Side Walls (SWs) are the continuation of the FW towards the back of the module.

Two actively cooled plates, named Caps, close the module on the top and on the bottom. On the backregion, the $30 \mathrm{~mm}$-thick Back Plate (BP) ensures the connection, mainly by the Tie Rods, of the module to the Back Supporting Structure (BSS). The combination of FW, SWs, Caps and BP is called Module Box (MB). 


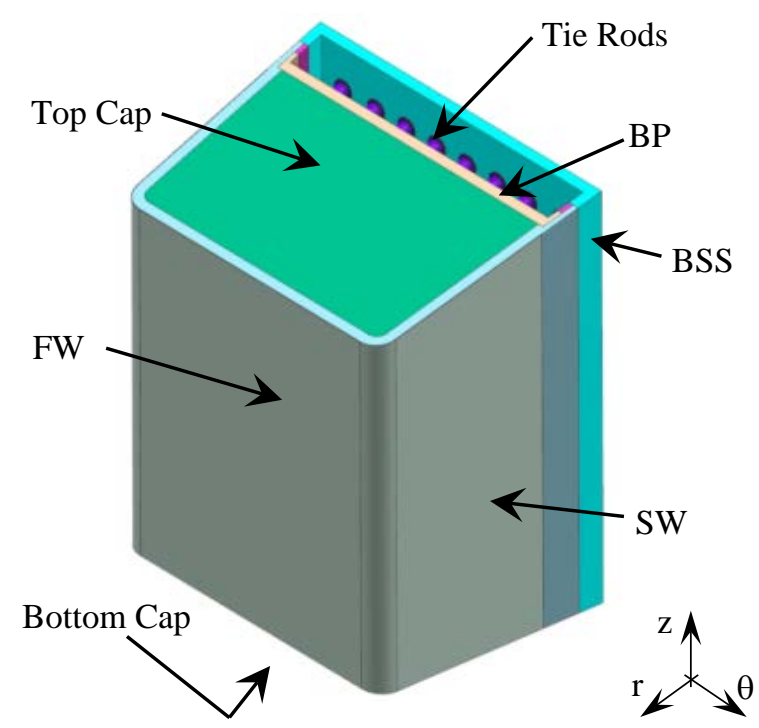

Fig. 1. Optimized Conservative HCLL OEM-externals.

As regards the internals (Fig. 2), a grid of $14 \mathrm{~mm}-$ thick radial-toroidal and radial-poloidal actively cooled plates, named horizontal and vertical Stiffening Plates (hSPs and vSPs) respectively, is mainly devoted to stiff the $\mathrm{MB}$ removing the nuclear heat power deposited into the breeder zone.

It has to be highlighted that MB plus SPs grid form a so called "beer box" structure, generating 80 Breeder Units (BUs) inside the module. Each one includes the breeder, the proper portion of SPs together with two 6.5 mm-thick Cooling Plates (CPs), devoted to improve the heat power removal.

Finally, between BUs and BP, a set of vertical plates has the main function to close the breeder zone and provide the Manifolds (MFs) for helium and breeder feeding.

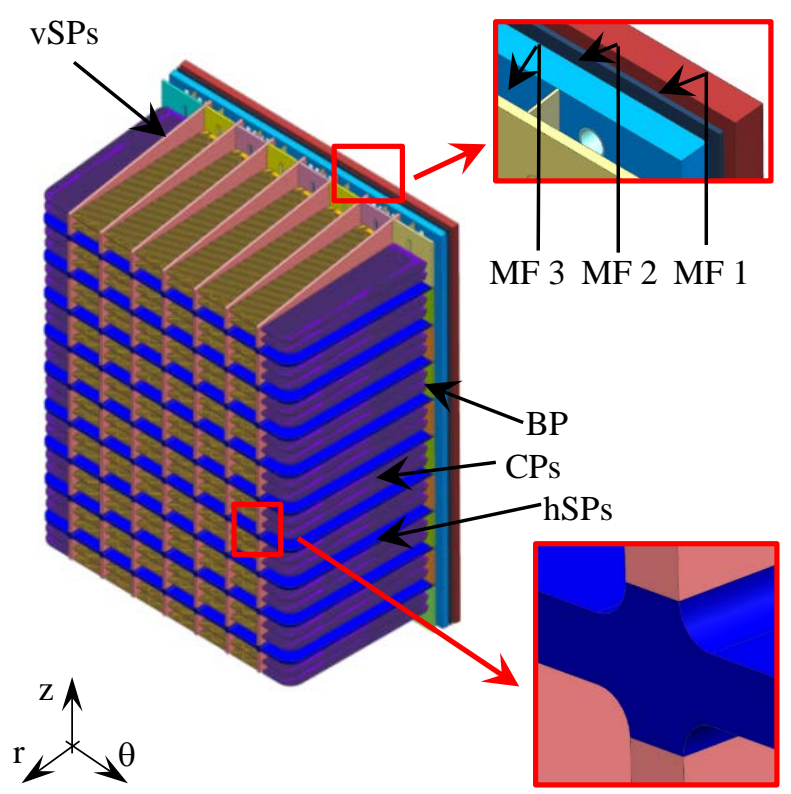

Fig. 2. Optimized Conservative HCLL OEM-internals.
In particular, MF 1 and MF 2 are devoted to house the helium during its flow in sequence among the actively-cooled components whereas MF 3 has the function to distribute the breeder inside the BUs (Fig. 3).

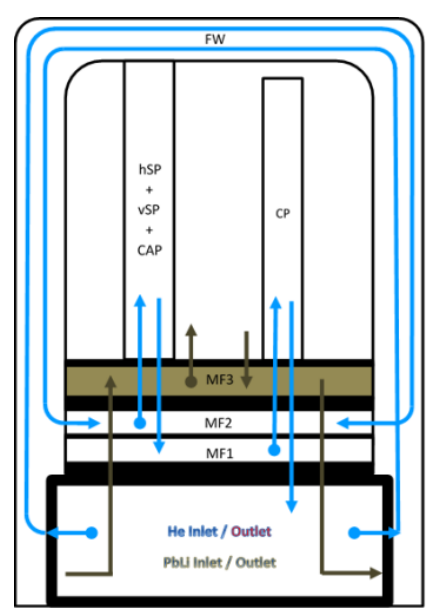

Fig. 3. Helium flow scheme.

\section{The FEM models}

In order to investigate thermal and thermomechanical performances of the "Optimized Conservative" DEMO HCLL OEM, paying also attention to analysis procedure automation, three successive FEM models (Model 1, Model 2 and Model 3) have been set-up. For all of them, cooling helium has been properly modelled, as well as the breeder.

As to material properties, they have been considered to depend uniquely by temperature [10].

\subsection{The geometric models and spatial discretization}

\section{Model 1}

In order to automate the analysis procedure, a simplified 3D model, reproducing a half radial-toroidal central slice of the HCLL OEM, has been set-up (Fig. 4). It includes the proper portion of FW-SW, tungsten layer, SPs, CPs, MFs plates, BP and Tie Rods together with corresponding breeder and helium.

It has to be noted that, due the odd number of FWSW cooling channels crossed by helium in countercurrent (Fig. 4), this model does not represent the basic geometric entity on which the HCLL design is based. Nevertheless, it has been set-up in order to automate the analysis procedure as it ensures reliable results to be obtained in a short time.

\section{Model 2}

In order to assess thermal and thermomechanical performances of the HCLL OEM, a model reproducing two adjacent half radial-toroidal central slices has been set-up (Fig. 4).

This model represents the basic geometric entity of HCLL as poloidal symmetry in the helium flow within FW-SW can be observed. 


\section{Model 3}

Finally, in order to assess the thermal and thermomechanical behaviour of the peripheral region of the HCLL OEM, a half-slice housing a Cap has been added to Model 2, so to obtain Model 3 (Fig. 4). It is to be noted that FW of the peripheral slice is equipped with only 7 cooling channels.

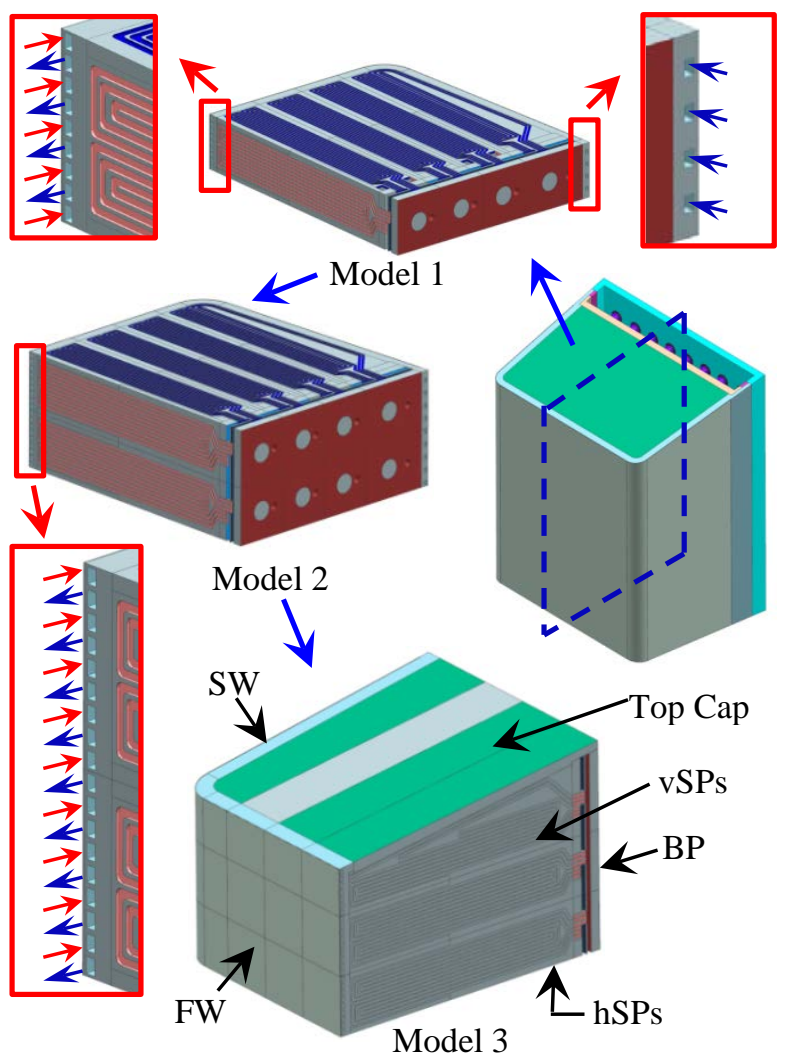

Fig. 4. Geometric layout of the models set-up.

A 3D tetrahedral mesh has been set-up for each model (Table 1). Linear elements have been used for thermal calculations, whereas quadratic ones have been adopted for thermomechanical analyses, where breeder and helium have not been considered as their effect has been simulated by imposing a proper pressure load.

Table 1. Mesh features summary.

\begin{tabular}{ccccc}
\hline Model & \multicolumn{2}{c}{ Th. analysis } & \multicolumn{2}{c}{ T.M. analysis } \\
\hline & Node & Elem & Node & Elem \\
\hline 1 & $202 \mathrm{k}$ & $1.1 \mathrm{M}$ & $1.2 \mathrm{M}$ & $656 \mathrm{k}$ \\
2 & $418 \mathrm{k}$ & $1.9 \mathrm{M}$ & $2.5 \mathrm{M}$ & $1.4 \mathrm{M}$ \\
3 & $611 \mathrm{k}$ & $2.7 \mathrm{M}$ & $3.7 \mathrm{M}$ & $2.1 \mathrm{M}$ \\
\hline
\end{tabular}

\subsection{Thermal loads and boundary conditions}

In order to investigate the thermal behaviour of the HCLL OEM under the DEMO steady state nominal scenario, a proper set of loads and boundary conditions has been adopted.

As to heat flux radiated from plasma, its nominal value $\left(0.5 \mathrm{MW} / \mathrm{m}^{2}\right)$ has been imposed on the FW straight section, whereas a decreasing value down to 0 , according a cosine law, has been assumed for the bend region. The radially non-uniform volumetric density of nuclear heat power deposited within steel and $\mathrm{Pb}-\mathrm{Li}$ by neutrons and gammas, are taken from [11].

Regarding the simulation of forced convective heat transfer occurring between helium and channels, the Gnielinski correlation [12] has been adopted for the heat transfer coefficients calculation. An inlet temperature of $300{ }^{\circ} \mathrm{C}$ has been imposed to helium entering from SW side (Fig. 4) whereas, as to the coolant which approaches the model from the FW central section (red arrows in Fig. 4), an inlet temperature equal to the average temperature of helium exiting from the same section has been imposed. Moreover, as helium exiting the FW-SW is mixed with the helium filling $M F$ 2, the inlet temperature of helium entering SPs has been calculated as MF 2 mixing temperature.

As to Model 3, this temperature has been imposed also to helium entering Cap (Fig. 3). Similarly, helium exiting SPs (and Cap in Model 3) is collected inside MF 1. Hence, CPs inlet temperature has been imposed to be equal to MF 1 mixing temperature.

Finally, only for Model 2, a thermal coupling condition has been imposed between model lower and upper surfaces in order to simulate model periodicity.

\subsection{Mechanical loads and boundary conditions}

In order to investigate the thermomechanical behaviour of the HCLL OEM under the DEMO steady state nominal scenario, a proper set of loads and boundary conditions has been adopted.

The pertinent thermal field resulting of the thermal calculation has been imposed, in the thermomechanical calculations in order to take into account the nonuniform thermal deformation field.

As to internal pressure, a load of $8 \mathrm{MPa}$ has been imposed onto all helium wetted surfaces, whereas pressure exerted by breeder on the module internals has been neglected.

Finally, in order to reproduce the connection to the BSS, radial displacement has been prevented to nodes lying on the BP external surface. Furthermore, poloidal and toroidal symmetry conditions have been imposed to nodes lying on the model boundary surfaces.

Moreover, in order to simulate the effect of the thermal expansion of the rest of the module, a thick shell has been supposed to be placed on the module top surface in Model 1 and 2. To this purpose, thermal field predicted on the model top surface has been projected on the shell.

\section{Analysis and results}

Un-coupled thermal and thermomechanical steady state analyses have been performed adopting the described FEM models. The most representative results are reported and critically discussed in the following. 


\subsection{Thermal results}

As far as thermal results are concerned, only those regarding Model 3 have been reported as it is the more realistic one among the models assessed in this study. In particular, the predicted temperature distribution within $\mathrm{MB}$ is depicted in Fig. 5. As it can be observed, the maximum temperature $\left(567^{\circ} \mathrm{C}\right)$ is slightly above the EUROFER limit of $550{ }^{\circ} \mathrm{C}$.

Anyway the region where temperature values exceed the prescribed limit is localized at the interface between FW and Cap (Fig. 6), due to the poor cooling of the FWCap interface region.

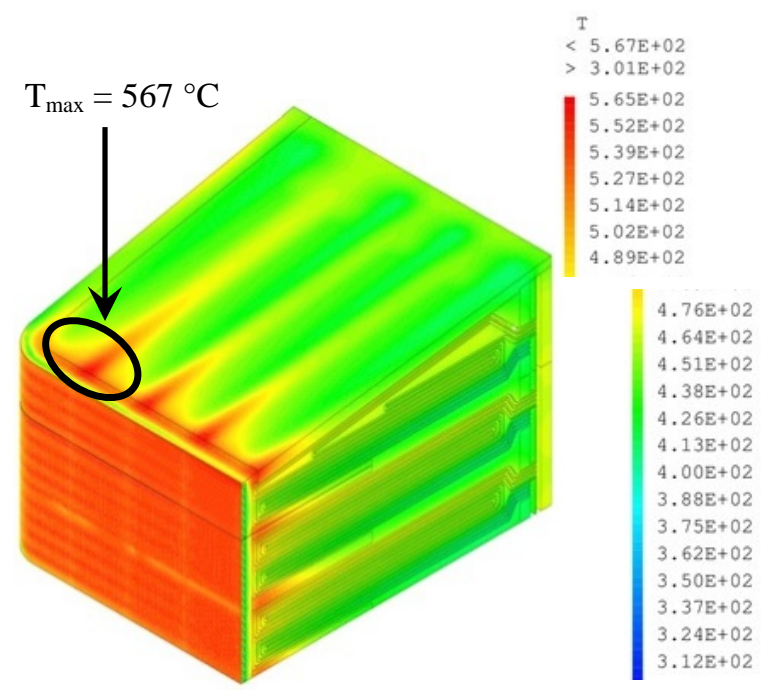

Fig. 5. Model 3 - MB thermal field.

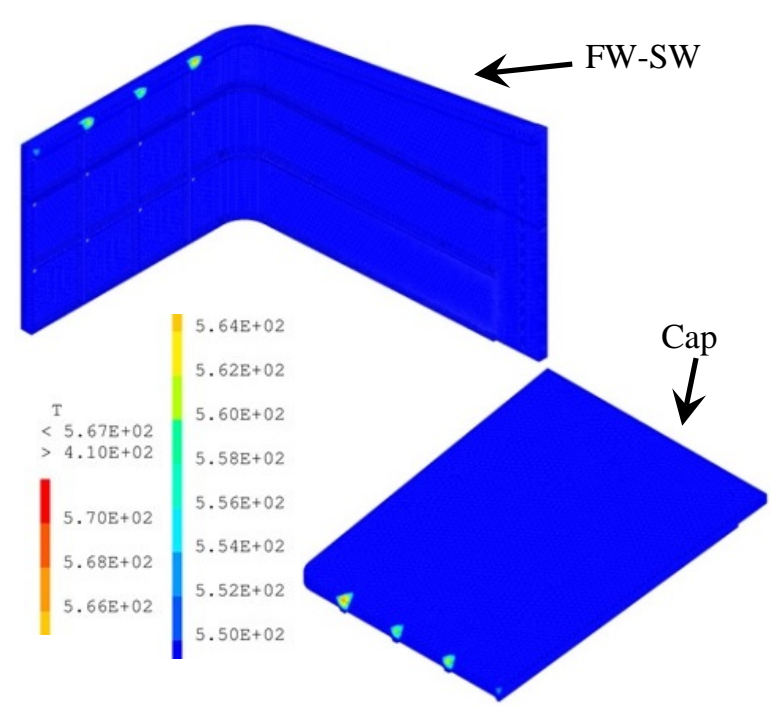

Fig. 6. Model 3 - MB regions exceeding $550{ }^{\circ} \mathrm{C}$.

Moreover, thermal field arising within SPs and CPs is shown in Figs. 7 and 8 respectively. It has to be noted that highest temperatures, around $550{ }^{\circ} \mathrm{C}$, are predicted within poorly cooled regions. Finally, thermal field within breeder and tungsten is reported in Fig. 9.

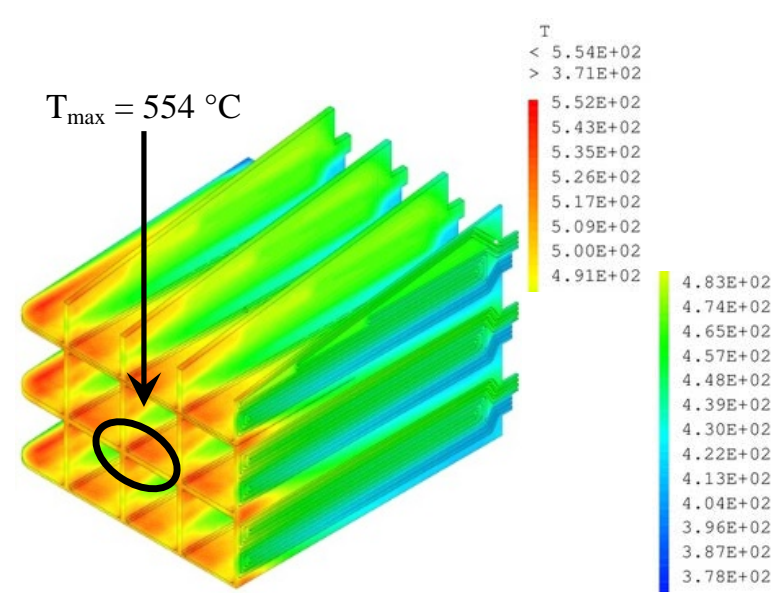

Fig. 7. Model 3 - SPs thermal field.

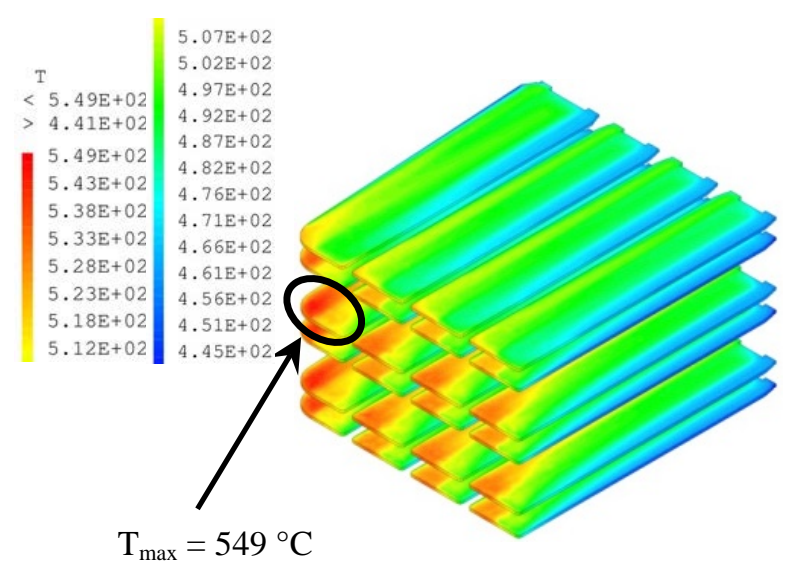

Fig. 8. Model 3 - CPs thermal field.

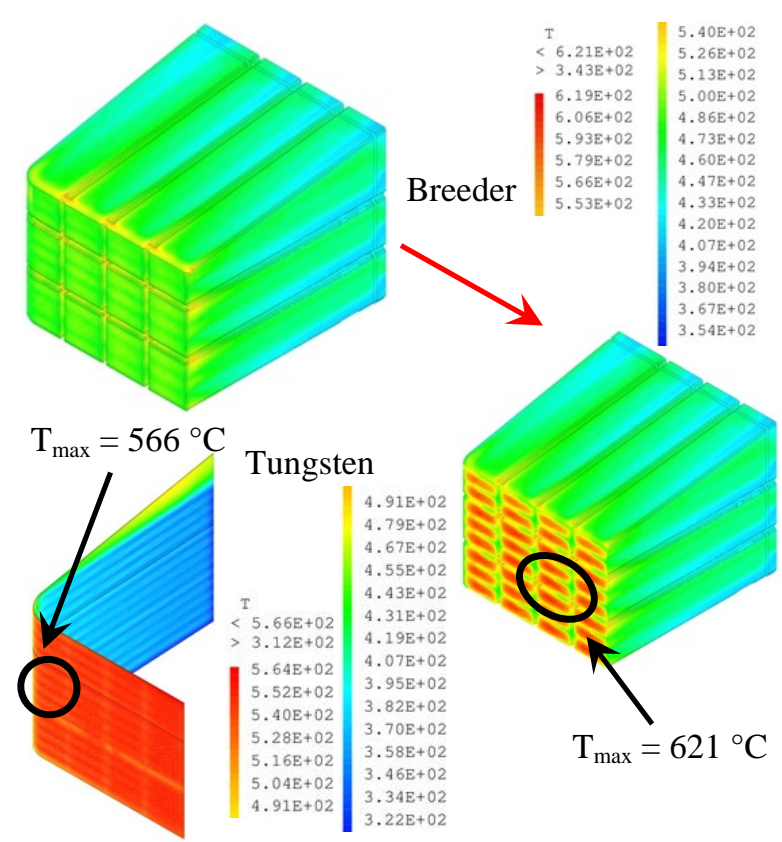

Fig. 9. Model 3 - Breeder and tungsten thermal field.

Regarding helium, the most significant outcomes are reported in Table 2. 
Table 2. Model 3 - Helium main outcomes.

\begin{tabular}{lcccc}
\hline & FW & SPs & Cap & CPs \\
\hline $\mathrm{T}_{\mathrm{av} \mathrm{in}}\left[{ }^{\circ} \mathrm{C}\right]$ & 300.0 & 406.6 & 465.2 \\
$\mathrm{~T}_{\mathrm{av} \mathrm{out}}\left[{ }^{\circ} \mathrm{C}\right]$ & 398.2 & 469.5 & 502.0 \\
$\mathrm{u}_{\mathrm{av}}[\mathrm{m} / \mathrm{s}]$ & 57.8 & 37.4 & 22.5 & 44.6 \\
$\mathrm{~h}_{\mathrm{av}}\left[\mathrm{W} / \mathrm{m}^{2}{ }^{\circ} \mathrm{C}\right]$ & 4542 & 3377 & 2144 & 4170 \\
$\Delta \mathrm{P}[\mathrm{MPa}]$ & 0.051 & 0.048 & 0.101 \\
\hline
\end{tabular}

\subsection{Thermomechanical results}

Regarding thermomechanical results, a comparison among FW deformed shapes ( isotropically amplified by a factor 50 ) of the 3 models (Fig. 10), allows to show the intense bending effect due to the thick shell imposed in Model 1 and 2 to simulate the effect of thermal expansion of the rest of the module.

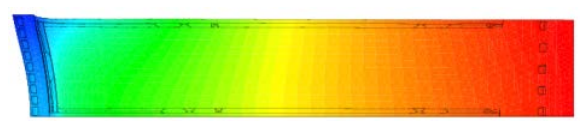

Model 1 x50
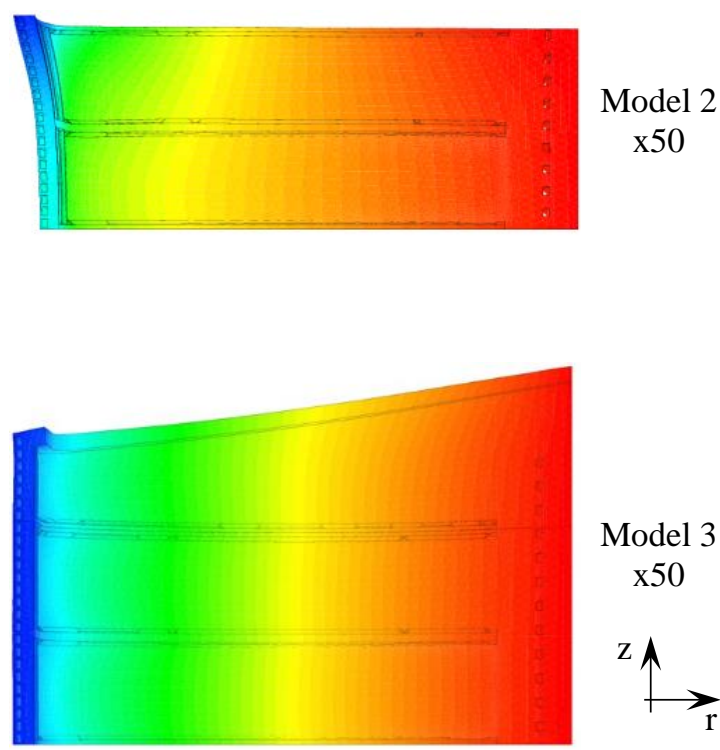

Model 3

$\mathrm{x} 50$

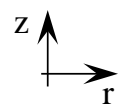

Fig. 10. Comparison among FW deformed shapes.

The shell imposition at the model top boundaries originates secondary stress within FW-SW top regions up to 1.5 greater than the corresponding regions of Model 3, causing the failure of RCC-MRx corresponding criteria.

Concerning Model 3 thermomechanical results, not affected by the shell boundary condition since this model includes the peripheral slice, the Von Mises stress field arising within MB is reported in Fig. 11.

As it can be observed, a wide $\mathrm{MB}$ region experiences Von Mises stress values lower than 500 MPa ( Fig. 11 a ) and, furthermore, outside FW-SW stress values are lower than $310 \mathrm{MPa}$ (Fig. $11 \mathrm{~b}$, printed out with an isotropic deformation amplification factor equal to 200 ).
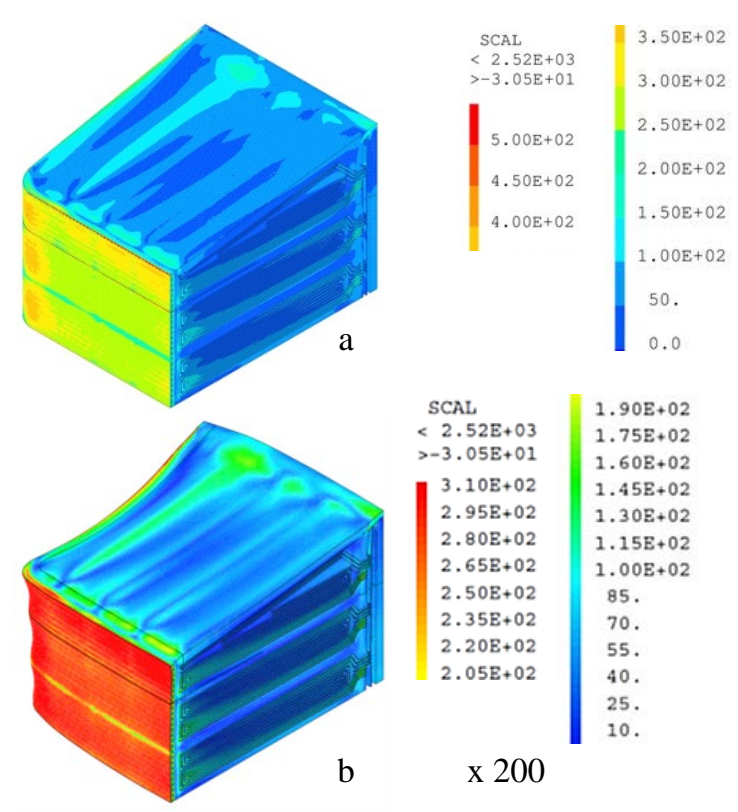

Fig. 11. Model 3 - MB Von Mises stress field.

Finally, a stress linearization procedure has been performed along lines shown in Fig. 12, at A and B toroidal heights, in order to verify the fulfilment of RCCMRx structural design criteria prescribed for nominal scenario, adopting the same methodology as for Advanced HCLL concept [6] with up to date stress limits [13]. Channels 1 and 5 of the lowest slice, together with Model 3 central channel, namely the fourth of the second central slice (Channel 13), and the central channel of the peripheral slice (Channel 21) have been considered.

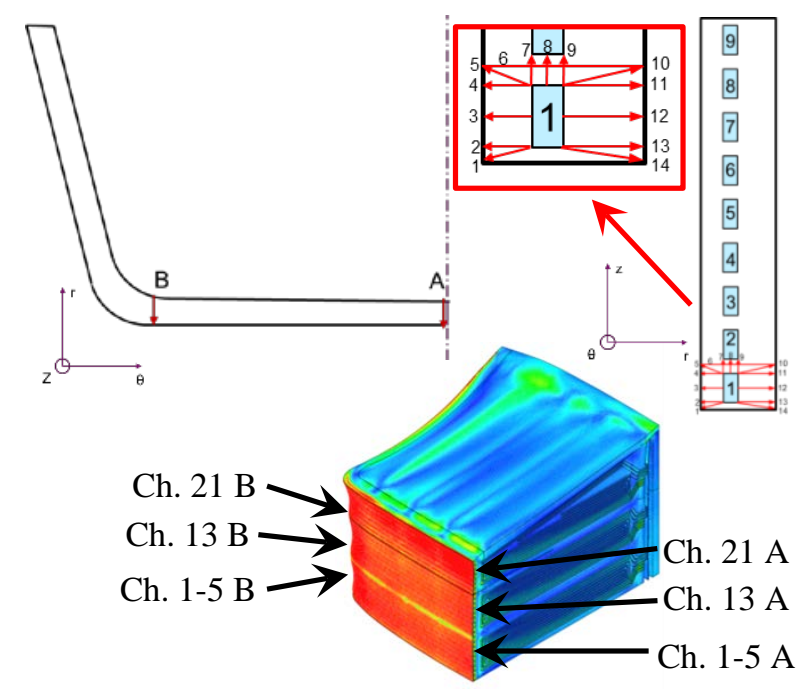

Fig. 12. Location of the stress linearization lines.

Results show that RCC-MRx design criteria have been fulfilled in all lines and channels of the toroidal height A, except for line 4 in Channel 21 (Fig. 13) where the two criteria involving secondary stress are not met. Nevertheless, looking at the trend lines, this may be due to a poor mesh in this region. As to the bend region (Fig. 
13) of the FW-SW (B toroidal height), only criterion against the risk of failure against immediate plastic flow localization because of the loss of ductility due to irradiation $\left(\mathrm{P}_{\mathrm{m}}+\mathrm{Q}_{\mathrm{m}} / \mathrm{S}_{\mathrm{em}}\right)$ is slightly above $1(\sim 1.1)$ for the lines from 2 to 5 , located in FW front thickness.

Channel 13A upper central slice

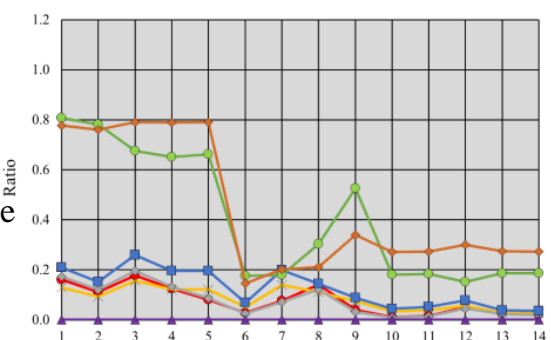

Channel 21A peripheral slice

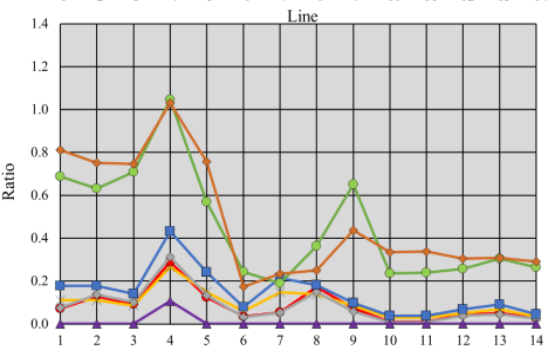

Channel 13B upper central slice

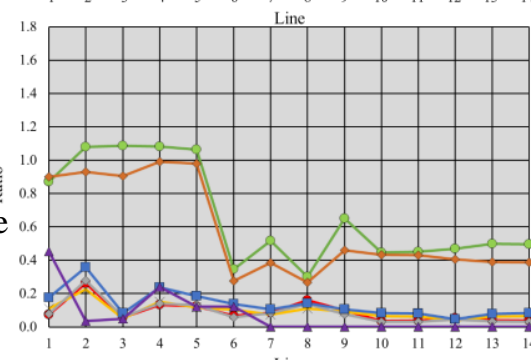

Channel 21B peripheral slice

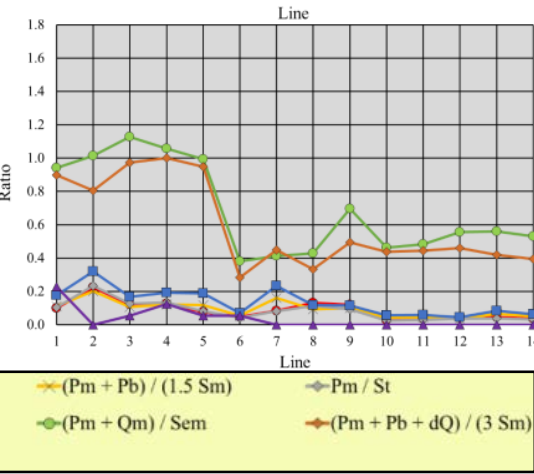

Fig. 13. Model 3 - Stress linearization results.

\section{Conclusion}

The research campaign, carried out within the framework of a close collaboration between CEA Saclay and University of Palermo, has allowed of minimizing the number of user-defined variables in the analysis procedure. Moreover, obtained results show good thermal and mechanical behaviour of the "Optimized Conservative” DEMO HCLL concept. However high temperature on the FW-Cap connection occurs and high stress is calculated near to the bend region of the FWSW, where only RCC-MRx criterion against immediate plastic flow localization because of the loss of ductility due to irradiation is slightly above the limit. Results suggest that, firstly, a more accurate mesh independence study is needed, adopting the FEM model in which the Cap is taken into account (Model 3), in order to carefully assess the influence on results of the spatial discretization grade. Secondly, some modifications of the module cooling scheme should be introduced in order to improve its thermal performances and, consequently, reduce the secondary stress amount, which is the main responsible of the failure in RCC-MRx criteria verification within FW-SW bend region.

\section{Acknowledgement}

This work has been carried out within the framework of the EUROfusion Consortium and has received funding from the Euratom research and training programme 2014-2018 under grant agreement No 633053. The views and opinions expressed herein do not necessarily reflect those of the European Commission.

\section{References}

[1] P. Chiovaro et al., Thermal-mechanical and thermalhydraulic integrated study of the Helium-Cooled Lithium Lead Test Blanket Module, Fus. Eng. and Des., vol. 85, (7-9), pp. 1147-1153, 2010.

[2] RCC-MRx, Design and Construction Rules for Mechanical Components of Nuclear Installations, AFCEN, 2013.

[3] Cast3M 2015. http://www-cast3M.cea.fr.

[4] Siemens NX v. 10.0. http: // www.plm.automation.siemens.com/en_us/products/nx/10/

[5] J. Aubert et al., Status of the EU DEMO HCLL breeding blanket design development and associated R\&D, this conference.

[6] J. Aubert, et al., Thermo-mechanical analyses and ways of optimization of the helium cooled DEMO First Wall under RCC-MRx rules, Fusion Eng. Des., DOI: 10.1016/j.fusengdes.2016.12.040, 2016.

[7] P. Arena, et al., Thermal optimization of the HeliumCooled Lithium Lead breeding zone layout design regarding TBR enhancement, Fusion Eng. Des., DOI: 10.1016/j.fusengdes.2017.03.086, 2017.

[8] R. Boullon et al., Investigation on the "advanced-plus" Helium Cooled Lithium Lead Breeding Blanket design concept for TBR enhancement regarding thermal and mechanical behaviour, this conference.

[9] G. Aiello, HCLL TBM design status and development, Fus. Eng. Des., Vol. 86, (9-11), Pages 2129-2134, 2011.

[10] F. Tavassoli, Present limits and improvements of structural materials for fusion reactors - a review. Journ. of Nucl. Mat., Vol. 302, pp. 73-88, 2002.

[11] J-C. Jaboulay et al., Nuclear Analysis of the HCLL “Advanced-plus” Breeding Blanket, this conference.

[12] V. Gnielinski, new equations for heat and mass transfer in turbulent pipe and channel flow, international chemical engineering, vol. 16, no.2, pp. 359-368 (1976).

[13] G. Aiello et al., Proposal of a set of design rules for the Blanket Conceptual Design in WPBB, https://idm.eurofusion.org/?uid=2MMPLE\&version=v1.0 